\title{
Kettle Hinders Cat, Shadow Does Not Hinder Shed: Activation of 'Almost Embedded' Words in Nonnative Listening
}

\author{
Mirjam Broersma ${ }^{1,2}$ \\ ${ }^{1}$ Max Planck Institute for Psycholinguistics, Nijmegen, The Netherlands \\ ${ }^{2}$ Nijmegen Institute for Cognition and Information, Radboud University Nijmegen, Nijmegen, The \\ Netherlands \\ m.broersma@nici.ru.nl
}

\begin{abstract}
A Cross-Modal Priming experiment investigated Dutch listeners' perception of English words. Target words were embedded in a carrier word (e.g., cat in catalogue) or 'almost embedded' in a carrier word except for a mismatch in the perceptually difficult $/ \mathfrak{x} / / \varepsilon /$ contrast (e.g., cat in kettle). Previous results showed a bias towards perception of $/ \varepsilon /$ over $/ æ /$. The present study shows that presentation of carrier

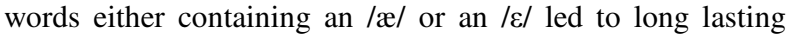
inhibition of embedded or 'almost embedded' words with an $/ æ /$, but not of words with an $/ \varepsilon /$. Thus, both catalogue and kettle hindered recognition of cat, whereas neither schedule nor shadow hindered recognition of shed.
\end{abstract}

Index Terms: spoken word recognition, nonnative listening, lexical activation, phonetic contrast

\section{Introduction}

When a listener finds it difficult to distinguish two sounds in a second language, this necessarily complicates the recognition of words containing these sounds. Minimal pairs differing in a difficult to distinguish contrast, like flash - flesh for Dutch listeners, are difficult to recognize, as hearing one word also leads to the activation of its unintended counterpart [1-4]. Similarly, partially overlapping onsets, like those of ACCIdent and EXEcute for Dutch listeners, temporarily lead to increased lexical activation of the unintended word for nonnative listeners, as compared to native listeners [5-7]. Further, non-words that are embedded in other words, like *lemp in eviL EMPire, cause more activation of real words (lamp) for nonnative listeners than for native listeners [2, 4, 8]. Thus, perceptual difficulties lead to the increased activation of unintended word forms in nonnative listening.

Cutler, Weber, and Otake propose that some phonemes may be more likely to cause such increased lexical activation than others. They showed that even when nonnative listeners distinguish two phoneme categories in their mental lexicon, at the level of phonetic processing, one category may be dominant. They argue that for Japanese listeners, English /r/ and /l/ are both perceived as /l/ [7], and that for Dutch listeners, English $/ \mathfrak{x} /$ and $/ \varepsilon /$ are both perceived as $/ \varepsilon /[5]$.

In line with this, Broersma [4] found a consistent and strong bias towards perception of $/ \varepsilon /$ over /æ/. As Dutch has only one phoneme in the phonetic space of English /æ/ and $/ \varepsilon /$, the contrast is perceptually difficult for Dutch listeners. Depending on the task, Dutch listeners have been found to categorize the contrast with a level of accuracy which was sometimes just [4] and sometimes amply above chance, but always less accurately than native listeners of English did.
Note that the bias towards perception of $/ \varepsilon /$ was not specific for nonnative (Dutch) listeners, but was found for native English listeners as well. In a series of six phonetic categorization experiments, both Dutch and English listeners had a similar bias towards "///" responses. A possible explanation might be that, according to the CELEX lexical database [9], the "/\&/" is more frequent than the "/æ/" in British English [4]. The bias towards perception of $/ \varepsilon /$ influenced the recognition of words as well, again for Dutch and English listeners alike. Listeners found it easier to recognize words with an $/ \varepsilon /$ than words with an $/ æ /$, regardless of whether the stimulus they had heard contained an /æ/ or an $/ \varepsilon /$. One auditory lexical decision experiment and two CrossModal Priming (CMP) experiments showed significantly higher percentages of "yes" responses and/or shorter reaction times (RTs) to words containing an $/ \varepsilon /$ than to words containing an /æ/. Thus, stimuli with an $/ \mathfrak{e} /$ or an $/ \varepsilon /$ activated

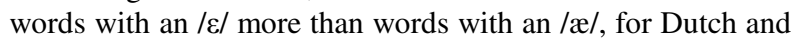
English listeners alike [4].

Although the bias towards $/ \varepsilon /$ perception may not be specific for the nonnative listeners in itself, in combination with these listeners' increased lexical activation of unintended words, the bias may lead to patterns of lexical activation that are specific for nonnative listeners. Although the English listeners may have an initial tendency to activate words with an $/ \varepsilon /$ more than words with an /æ/, eventually they will recognize the intended word and efficiently deactivate its competitor. For the nonnative listeners, this spurious lexical activation may not be undone as efficiently and therefore the bias towards $/ \varepsilon /$ perception may have a larger and longer lasting effect on word recognition.

The present study therefore investigates whether there is an symmetry in Dutch listeners' activation of words with an $/ \mathfrak{x} /$ and words with an $/ \varepsilon /$, for words that are embedded or 'almost embedded' (mismatching the input in the $/ æ /-/ \varepsilon /$ contrast) in the onset of a carrier word. It investigates whether the recognition of words containing an /æ/ suffers more from the activation of lexical competitors than the recognition of words containing an $/ \varepsilon /$.

In a CMP experiment, Dutch listeners were presented with words like cattle and schedule, containing the embedded words cat and shed, and with kettle and shadow that, apart

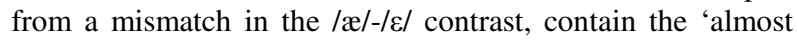
embedded' words cat and shed. Carrier words were used as auditory primes, and (almost) embedded words as visual targets. The auditory primes were expected to activate the intended (carrier) words more strongly than the unintended (embedded and 'almost embedded') words, if only on the basis of the non-ambiguous second part of the stimulus. Indeed, whereas a carrier word may initially activate both the intended word and the embedded word, after presentation of 
the disambiguating part of the stimulus, activation of the embedded word is usually found to disappear [10].

Previous results from a similar experiment, but with items differing in consonant voicing [11], showed that no activation was found for embedded words (precedent - press) or for 'almost embedded' words that mismatched the stimulus in the voicing of the final consonant (president - press). Presumably, by the end of the carrier word, the intended word had been recognized and the lexical competition between the intended word and the (almost) embedded word had been resolved. Note that the obstruent voicing contrasts exists in Dutch, but are not distinctive in word-final position. Although Dutch listeners have been found to categorize the word-final contrasts with a native like level of accuracy [4, 12], minimal pairs and non-words mismatching in the word-final voicing contrast do cause spurious lexical activation for Dutch listeners, compared to native English listeners $[2,4]$. In the case of the (almost) embedded words, it was the presence of a longer and better matching lexical competitor which led to the deactivation of the (almost) embedded words. Thus, no activation of press was found after presentation of either precedent or president, due to the competition from these carrier words themselves.

Similarly, even if the Dutch listeners do not recognize the $/ æ /-/ \varepsilon /$ contrast accurately, the activation of the carrier word, matching a larger portion of the input than an (almost) embedded word does, might lead to the recognition of the carrier word and to the deactivation of the (almost) embedded word. In that case, after presentation of the carrier word, no effect of the carrier word on the recognition of the embedded or 'almost embedded' word should be noticeable when it is presented as a visual target word.

On the other hand, the bias towards $/ \varepsilon /$ perception may lead to the relatively strong inhibition of lexical competitors containing an $/ æ /$, and to less inhibition of lexical competitors containing an $/ \varepsilon /$. Thus, after hearing catalogue as well as after hearing kettle, the activation of the (almost) embedded word cat may be strongly suppressed, whereas after hearing schedule as well as after hearing shadow, the activation of the (almost) embedded word shed may not be suppressed as strongly. In that case, the presentation of the carrier word may influence the subsequent recognition of visual target words with an $/ æ /$ more than that of words with an $/ \varepsilon /$.

The consonant items used in [11], matching or mismatching the carriers in final consonant voicing, are used again in the present experiment, and are compared to words matching or mismatching the carriers in the $/ æ /-/ \varepsilon /$ contrast.

\section{Method}

\subsection{Participants}

Participants were 36 native speakers of Dutch, volunteers, who were recruited at Nijmegen University. They were proficient in English as a second language and had received on average 7.3 years of English instruction $(\mathrm{SD}=1.5)$. None of them reported any hearing loss, visual loss or reading disability.

\subsection{Materials}

Experimental items (visual target words and auditory primes) are presented in Table 1. As experimental items, 24 monosyllabic English words were selected as visual targets, 12 for the consonant manipulation and 12 for the vowel manipulation. Of the consonant items, half ended with a voiced obstruent (/b,d,v,z/) and half with a voiceless obstruent $(/ \mathrm{t}, \mathrm{s} /)$, and of the vowel items, half contained an $/ æ /$ and half an $/ \varepsilon /$. For each of these target words, three words were selected as auditory primes. First, for the Identity condition, a di- or trisyllabic carrier word was found in which the target word occurred as an initial embedding (e.g., catalogue for cat, schedule for shed). Second, for the Mismatch condition, a carrier word was found in which the target word was 'nearly embedded', apart from a mismatch in final consonant voicing, or in the $/ æ /-/ \varepsilon /$ contrast. Thus, for each consonant target the voicing of the final consonant was reversed, and for each vowel target $/ \mathfrak{a} /$ and $/ \varepsilon /$ were reversed, and a word was found that contained the resulting monosyllable as an initial embedding (e.g., kettle for cat, shadow for shed). Third, for the Control condition, a phonologically and semantically unrelated di- or trisyllabic word was selected (e.g., pocket for cat, virgin for shed). The experimental stimuli, but not the fillers, are the same as those used in [11] (Experiment 2).

Table 1. Experimental items.

\begin{tabular}{|l|l|l|l|}
\hline $\begin{array}{l}\text { Target } \\
\text { word }\end{array}$ & $\begin{array}{l}\text { Identity } \\
\text { prime }\end{array}$ & $\begin{array}{l}\text { Mismatch } \\
\text { prime }\end{array}$ & $\begin{array}{l}\text { Control } \\
\text { prime }\end{array}$ \\
\hline Voiced consonants \\
\hline Add & Addict & Attitude & Limousine \\
Bud & Budget & Butter & Hydrant \\
Cab & Cabinet & Capital & Balustrade \\
Card & Cardinal & Carton & Orange \\
Ease & Easel & Easter & Silver \\
Serve & Survey & Surface & Vacancy \\
\hline Voiceless consonants & \\
\hline Art & Article & Ardent & Subsidize \\
Bliss & Blister & Blizzard & Steady \\
Crate & Crater & Cradle & Exit \\
Debt & Detonate & Dedicate & Certify \\
Press & Precedent & President & Vegetate \\
State & Status & Stadium & Hazel \\
\hline /æ/ & \multicolumn{5}{|l}{} \\
\hline Cat & Catalogue & Kettle & Pocket \\
Damn & Damage & Democrat & Albatross \\
Lamb & Laminate & Lemon & Balance \\
Pant & Pantomime & Pentagram & Synthesize \\
Tack & Tactic & Textile & Finger \\
Tan & Tantrum & Tentacle & Bulletin \\
\hline /\&/ & \multicolumn{5}{|l}{} \\
\hline Chess & Chestnut & Chastity & Anarchism \\
Deaf & Definite & Daffodil & Hovercraft \\
Dress & Dressage & Drastic & Stupid \\
Edge & Educate & Agile & Whistle \\
Egg & Egotist & Agony & Lunatic \\
Shed & Schedule & Shadow & Virgin \\
\hline
\end{tabular}

As fillers, 72 words and 96 non-words were selected. Each filler was combined with one auditory prime word. For 8 of the non-words, Identity primes were selected that contained the non-word as an initial embedding, and for 8 non-words Mismatch carriers were selected, that contained the non-word except for a mismatch in the vowel (for 4 items) or in the final consonant (for 4 items). For all other filler words and nonwords, a phonologically and semantically unrelated word was selected as auditory prime.

All visual targets were monosyllabic, and half of the auditory primes were disyllabic and the other half trisyllabic words. Stimuli were not spelled or pronounced like existing Dutch words. The prime words were recorded by a male 
native speaker of British English in a soundproof booth using a high quality microphone onto digital audiotape and downsampled to $16 \mathrm{kHz}$ during transfer to a computer. The speaker read the items one by one, separated by a pause, in a clear citation style. Stimuli were extracted from the sound file using the speech editor Xwaves.

\subsection{Design}

The experimental items were divided over three lists, with the voiced consonant, voiceless consonant, /æ/, and $/ \varepsilon /$ items distributed evenly over the three conditions. Each list also contained all of the filler words and filler non-words, so that each participant was presented with 96 words (including 24 experimental words and 72 filler words) and 96 non-words. Of these items, 8 experimental target words and 8 filler nonwords were preceded by an Identity prime and the same number of items by a Mismatch prime, and all other items by unrelated Control primes. Items were presented in a semirandom order, such that experimental targets did not directly follow one another and no more than five words or non-words were shown in succession.

\subsection{Procedure}

Participants were tested one at a time in a quiet room. They received written instructions in their native language, informing them that on each trial they would hear an English word, directly after which an English word or non-word would appear on a computer screen. They were asked to decide whether the visual stimulus was an English word or not, and to indicate their decision by button press, both as fast and as accurately as possible. The auditory prime words were presented binaurally over closed headphones at a comfortable listening level and the visual stimuli were presented in large font on a computer screen in front of the participants, directly at offset of the auditory prime. Participants responded by pressing one of two response buttons, labeled "yes" and "no". No time limit was imposed for the responses. After each button press, the next trial started. The experiment started with 12 practice trials and was controlled with Nijmegen Experiment Set-Up software.

\section{Results}

Proportions of correct ("yes") responses and reaction times (RTs) for the correct responses are presented in Table 2. Reaction times were measured from the offset of the auditory prime, equaling the onset of the presentation of the visual target. Outliers, with RTs longer than $1300 \mathrm{~ms}$, were removed. There were no significant effects in the analysis of arcsine-transformed proportions of correct responses.

For the RTs, ANOVAs were done with the mean reaction time of the correct responses as dependent variable. Due to a large number of empty cells, Univariate ANOVAs were performed instead of Repeated Measures in the analysis by subjects (F1). Figure 1 shows the amount of priming and inhibition in the Identity and Mismatch condition, compared to the Control condition.

First, the results for the consonant items replicate those of Broersma [11]. For the consonant items, there was no effect of Condition $(F 1(2,196)<1 ; F 2(2,20)<1)$. Thus, hearing a carrier word did not influence the recognition of an embedded word or a nearly embedded word. There were no significant effects of, or interactions involving, Voicing (voiced - voiceless final consonants).
Table 2. Percentage correct and reaction times of correct responses.

\begin{tabular}{|l|l|l|}
\hline Condition & \% Correct & RT (ms) \\
\hline Consonants \\
\hline Control & 87 & 711 \\
Identity & 89 & 717 \\
Mismatch & 82 & 730 \\
\hline /a/ \\
\hline Control & 68 & 640 \\
Identity & 75 & 714 \\
Mismatch & 65 & 724 \\
\hline /\&/ & 707 \\
\hline Control & 88 & 675 \\
Identity & 87 & 679 \\
Mismatch & 95 &
\end{tabular}

Crucially, for the vowel items, as Figure 1 shows, the pattern of results differed for target words with an $/ \mathfrak{a} /$ and for those with an $/ \varepsilon /$. Indeed, there was a significant interaction between Condition and Phoneme $(/ æ /-/ \varepsilon /)(F 1(2,180)=$ $3.09, p<.05 ; F 2(2,20)=5.06, p<.05)$.

For the target words with an $/ æ /$, the effect of condition just missed significance $(F 1(2,80)=3.10, p=.051 ; F 2(2$, $10)=5.73, p<.05)$. For these words, there was inhibition in the Identity condition $(F 1(1,55)=4.92, p<.05 ; F 2(1,5)=$ $9.85, p<.05)$ as well as in the Mismatch condition $(F 1$ (1, $50)=5.53, p<.05 ; F 2(1,5)=9.72, p<.05)$, and the amount of inhibition in the Identity and the Mismatch conditions did not differ $(F 1(1,55)<1 ; F 2(1,5)<1)$. For the target words with an $/ \varepsilon /$, on the other hand, there was no significant effect of condition $(F 1(2,100)<1 ; F 2(2,10)<1)$.

Thus, for the target words with an /æ/, recognition of the target word was hindered when it was preceded by an Identity prime or by a Mismatch prime (compared to a Control prime). For target words with an $/ \varepsilon /$, on the other hand, auditory primes did not affect the recognition of the visual target words, similarly to the consonant target words.

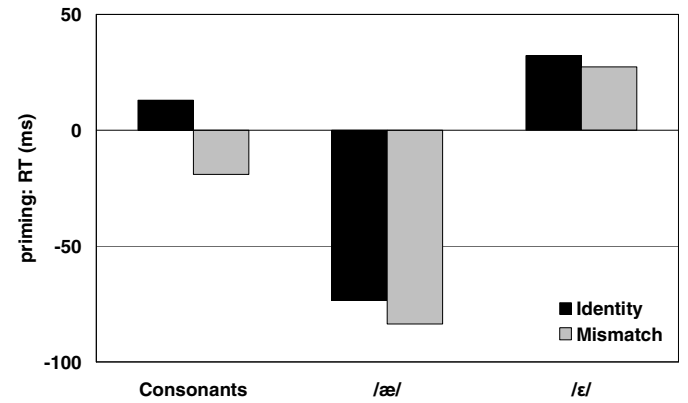

Figure 1: Priming, computed as the difference between the reaction times of the correct responses in the Identity or the Mismatch condition and the Control condition, with a negative value indicating inhibition.

\section{Discussion}

For the consonant items, the activation of longer and better matching carrier words was expected to deactivate the (shorter) embedded words and the (shorter as well as mismatching) 'almost embedded' words, which should lead to the absence of any effect of Identity or Mismatch primes on the recognition of visual target words. Indeed, Identity or 
Mismatch primes did not affect the recognition of the target words, replicating the results of [11]. Presumably, the activation of the carrier words deactivated the embedded and the 'almost embedded' words for the consonant items.

Similar results were found for the target words containing an $/ \varepsilon /$. There was no effect of the Identity and Mismatch primes on the recognition of the target words, and thus there was no evidence of any remaining lexical activation of (almost) embedded words at offset of the carrier words for the target words containing an $/ \varepsilon /$.

However, the results show an asymmetry in the activation of embedded and 'almost embedded' words with an $/ \varepsilon /$ and those with an /æ/. For the target words with an /æ/, inhibition was found both in the Identity condition and in the Mismatch condition. Presumably, the perceptual bias towards recognition of $/ \varepsilon /$ led to a long lasting inhibition of words containing an /æ/, even when they matched the speech input only temporarily and, for the 'almost embedded' words, only partially. Thus, recognition of the word shed was never hindered, neither by presentation of schedule, nor shadow, but recognition of the word cat, on the other hand, was hindered both by presentation of catalogue and kettle.

For target words with an $/ \varepsilon /$, the auditory stimulus may have activated the embedded and the 'almost embedded' words initially, but after the presentation of the full word form, no evidence for this activation remained. The results for the target words with an $/ \varepsilon /$ were similar to the results for the consonant items, and similar results were also found for the same consonant items in a previous study, for Dutch as well as for English listeners [11]. Thus, the Dutch listeners seem to recognize the target words with an $/ \varepsilon /$ efficiently and in a native-like manner.

The recognition of target words with an /æ/ was hindered both by the presentation of carrier words actually containing the target word, and by presentation of carrier words almost containing the target word. After hearing either catalogue or kettle, the activation of cat was suppressed so much that this word's recognition was delayed, even when it was presented at offset of the carrier word, by which time any embedded competitors are normally no longer active [10].

Note that the results do not mean that carrier words with an /æ/ activate 'almost embedded' words with an $/ \varepsilon /$, whereas carrier words with an $/ \varepsilon /$ do not activate 'almost embedded' words with an $/ æ /$. Such an asymmetry would entail a difference between the Mismatch condition and the Control condition for the target words with an /E/ (with shadow activating shed), and no difference between the Mismatch and the Control condition for the target words with an /æ/ (with kettle not activating cat), both contrary to the actual results. The results rather show that words containing an /æ/ suffer more from inhibition from competing carrier words than words containing an $/ \varepsilon /$.

The results are thus in line with several previous experiments, that did not show such unidirectional spurious lexical activation either [2, 4, 6]. One auditory lexical decision experiment and four CMP experiments showed that spurious lexical activation for the Dutch listeners occurred in both directions: stimuli with an /æ/ activated words with an $/ \varepsilon /$, but stimuli with an $/ \varepsilon /$ activated words with an $/ æ /$ as well. Thus, flash activated flesh as much as vice versa [2,4], the onsets of accident and execute activated both words to the same extent [6], and *ket activated cat as much as *chas activated chess $[2,4]$. Also in line with the present results however, those studies showed that Dutch listeners found it generally easier to recognize words with an $/ \varepsilon /$ than words

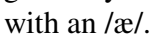

Note also that the present experiment does not assess the occurrence of the activation of (almost) embedded words that may occur during unfolding of the carrier word over time, but rather the state of affairs at offset of the carrier word.

The present results show that the bias towards perception of $/ \varepsilon / 4$ ] led to long lasting inhibition of lexical competitors containing an /æ/, which hindered the recognition of those words even after offset of the carrier words, and to the absence of such long lasting inhibition of lexical competitors containing an $/ \varepsilon /$. This made the recognition of words with an $/ æ /$ more difficult for Dutch listeners than the recognition of words with an $/ \varepsilon /$.

\section{References}

[1] Pallier, C., Colomé, A., and Sebastián-Gallés, N. "The influence of native-language phonology on lexical access: Exemplar-based versus abstract lexical entries", Psychological Science, 12: 445-449, 2001.

[2] Broersma, M., Comprehension of non-native speech: Inaccurate phoneme processing and activation of lexical competitors [oral], Proceedings of the 7th International Conference on Spoken Language Processing (pp. 261264), Center for Spoken Language Research, University of Colorado, Boulder (CD-ROM), 2002.

[3] Cutler, A. and Otake, T., Pseudo-homophony in nonnative listening, Poster presented at the 75th meeting of the Acoustical Society of America, New York, 2004.

[4] Broersma, M., Phonetic and Lexical Processing in a Second Language, Radboud University Nijmegen, Nijmegen, The Netherlands, 2005.

[5] Weber, A. and Cutler, A. "Lexical competition in nonnative spoken-word recognition", Journal of Memory and Language, 50: 1-25, 2004.

[6] Broersma, M., Accident - execute: Increased activation in nonnative listening [oral], Proceedings of the 9th International Conference on Spoken Language Processing, Pittsburgh, PA (CD-ROM), 1519-1522, 2006.

[7] Cutler, A., Weber, A., and Otake, T. "Asymmetric mapping from phonetic to lexical representations in second-language listening", Journal of Phonetics, 34: 269-284, 2006.

[8] Sebastián-Gallés, N., Echeverría, S., and Bosch, L. "The influence of initial exposure on lexical representation: Comparing early and simultaneous bilinguals", Journal of Memory and Language, 52: 240-255, 2005.

[9] Baayen, H., Piepenbrock, R., and Gulikers, L., The CELEX Lexical Database (CD-ROM), Linguistic Data Consortium, University of Pennsylvania, Philadelphia, PA, 1995.

[10] Salverda, A. P., Dahan, D., and McQueen, J. M. "The role of prosodic boundaries in the resolution of lexical embedding in speech comprehension", Cognition, 90: 51-89, 2003.

[11] Broersma, M., 'President' does not excite 'press': The limits of spurious lexical activation in L2 listening, 16th International Congress of Phonetic Sciences (ICPhS), Saarbrücken, Germany, August 6-10, 2007, submitted.

[12] Broersma, M. "Perception of familiar contrasts in unfamiliar positions", Journal of the Acoustical Society of America, 117: 3890-3901, 2005. 